\title{
Beitrag der Migranten zur Entwicklung : Wirtschaftsperspektive
}

\section{Philippe Wanner}

\section{(2) OpenEdition \\ 1 Journals}

Electronic version

URL: http://journals.openedition.org/sjep/332

DOI: $10.4000 /$ sjep.332

ISSN: 1663-9677

Publisher

Institut de hautes études internationales et du développement

Printed version

Date of publication: 1 décembre 2008

Number of pages: 129-140

ISBN: 978-2-940415-08-3

ISSN: 1660-5926

\section{Electronic reference}

Philippe Wanner, «Beitrag der Migranten zur Entwicklung: Wirtschaftsperspektive », Schweizerisches Jahrbuch für Entwicklungspolitik [Online], 27-2 | 2008, Online erschienen am: 31 Mai 2010, abgerufen am 08 September 2020. URL : http://journals.openedition.org/sjep/332 ; DOI : https://doi.org/ $10.4000 /$ sjep.332 


\section{Beitrag der Migranten zur Entwicklung: Wirtschaftsperspektive}

Philippe Wanner*

\section{Einführung}

Die Frage, welchen Beitrag die Migration zur wirtschaftlichen und sozialen Entwicklung der Länder des Südens leisten kann, wurde wiederholt diskutiert, aber ein echter Konsens zeichnet sich bis heute nicht $a b^{1}$. Während einige Autoren die Verarmung durch den sogenannten brain drain - das Abfliessen von Kompetenzen - unterstreichen, sehen andere in der Migration eine Chance für die Länder des Südens, da Migrationsbewegungen stets mit einem Rückfluss an Reichtum und Kompetenzen in das Herkunftsland begleitet seien. Damit kommt der volumenmässigen Abschätzung der Rücküberweisungen von Migrantinnen und Migranten in ihre Heimat - der sogenannten Remittances - und ihrer wirtschaftlichen Bedeutung bei der Betrachtung der Wechselwirkung zwischen Migration und Entwicklung eine zentrale Bedeutung zu. Diese Transfers werden im Folgenden näher untersucht.

Dass die Rücküberweisungen nicht nur Einzelpersonen und Haushalten, sondern der Wirtschaft als Ganzes zugutekommen, wird in der Regel nicht bestritten. Allerdings lassen sich in verschiedenen Kontexten Verzerrungen beobachten, die von diesen Transfers hervorgerufen werden. Einige der spezifischen Problemstellungen im Zusammenhang mit diesen Geldflüssen, aber auch ihre positiven Wirkungen, werden weiter unten hervorgehoben. Zudem muss auch der Frage nachgegangen werden, ob die Verwendung dieser Gelder dahingehend optimiert werden kann, dass sie ihr maximales ökonomisches Potenzial entfalten. Als Einstieg werden zunächst verschiedene Kennzahlen präsentiert, die den Umfang der Rücküberweisungsflüsse veranschaulichen. Erörtert werden zunächst auch die Determinanten für diese Rücküberweisungen, die genutzten Kanäle und die Konsequenzen der Transfers, um anschliessend die spezifischen Beziehungen zwischen Remittances und wirtschaftlicher Entwicklung zu diskutieren.

\section{Umfang der Geldflüsse zwischen Nord und Süd}

Die Rücküberweisungen haben sich im Gleichschritt mit der Zunahme der modernen Migrationsbewegungen entwickelt. Dies erklärt, weshalb die Problematik seit mehreren Jahrzehnten Gegenstand wissenschaftlicher Untersuchungen ist. Ins Blickfeld der Öffentlichkeit gelangte die Frage indessen erst, als sich

* Professor am Laboratoire de démographie et d'études familiales, Faculté des sciences économiques et sociales, Universität Genf.

1 Siehe zum Beispiel R. Faini, „Migrations et transferts de fonds. Impact sur les pays d'origine“, Revue d'économie du développement, Bd. 21, Nr. 2-3, 2007, S. 153-182. 
angesichts des Umfangs der Geldströme auch internationale Organe wie beispielsweise die Organisation für wirtschaftliche Zusammenarbeit und Entwicklung (OECD), der Internationale Währungsfonds (IWF), die Internationale Organisation für Migration (IOM) oder die Weltbank dafür zu interessieren begannen. Grund für das zunehmende Interesse an den Transfers ist nicht nur deren wachsende Bedeutung, sondern auch das Auftauchen verschiedener damit verbundener Fragen, die im Folgenden angesprochen werden.

In der Tat ist der Umfang der Rücküberweisungen heute im Begriff, das Volumen der öffentlichen Entwicklungshilfe zu übertreffen. Dies bedeutet indes nicht, dass sie die öffentliche Hilfe ersetzen. Vielmehr stellen sie für zahlreiche Empfängerländer eine zunehmend wichtige Kapitalquelle dar, die von der wirtschaftlichen Konjunktur kaum beeinflusst wird. Eine weitere Besonderheit der Rücküberweisungen ist, dass sie einer Vielzahl von Empfängern zugutekommen: Einer Schätzung des Internationalen Fonds für landwirtschaftliche Entwicklung (IFAD) zufolge kommt weltweit eine von zehn Personen in den Genuss solcher Transfers ${ }^{2}$.

Insgesamt jedoch ist das Phänomen der Rücküberweisungen kaum erforscht und nur unzulänglich beschrieben. Zwar werden die Instrumente zur Messung der Finanzströme zwischen den Ländern ständig weiterentwickelt, aber verlässliche Schätzungen des Gesamtumfangs der Geld- und Gütertransfers von Migrantinnen und Migranten in ihre Heimat liegen bis heute nicht vor. Dies liegt vor allem daran, dass sich das Konzept der Remittances nur schwer griffig definieren lässt. Remittances stammen von Personen ausländischer Herkunft, deren Status sehr unterschiedlich sein kann: Migrantinnen und Migranten gemäss offizieller Definition der Vereinten Nationen (die bereits seit mindestens einem Jahr im Gastland wohnhaft sind oder die bereits vor der Auswanderung die Absicht haben, mindestens ein Jahr in einem anderen als in ihrem Wohnsitzstaat zu leben), Kurzzeitmigrantinnen und -migranten (die meist als Saisonarbeiter im Gaststaat leben), illegal im Gaststaat lebende Personen, im Gastland geborene Personen ausländischer Herkunft, die nach wie vor über Bindungen zur Heimat verfügen, und in gewissen Ländern gar Grenzgängerinnen und Grenzgänger ${ }^{3}$. Auch die Herkunft der überwiesenen Gelder ist äusserst vielfältig und reicht von regelmässig ersparten und in die Heimat überwiesenen Beträgen über Summen, die zum Zeitpunkt der Rückkehr in die Heimat transferiert werden, bis hin zu Sozialtransfers nach dem Ende der Migration. Die Erfassung der Remittances wird dadurch noch erschwert. Aus dem Blickwinkel der Entwicklungsproblematik müsste die Gesamtheit der transferierten Beträge berücksichtigt werden, denn nur so lässt

2 International Fund for Agricultural Development (IFAD), Sending Money Home, Rome, IFAD, Dezember 2007, <http://www.ifad.org/events/remittances/maps/brochure.pdf>

3 Gemäss offizieller Definition handelt es sich bei Rücküberweisungen um regelmässige Sendungen von erwerbstätigen Migrantinnen und Migranten, aber auch um Gehälter von Arbeitnehmerinnen und -nehmern, die während mindestens zwölf Monaten im Ausland wohnhaft sind, sowie um Geldtransfers von Migrantinnen und Migranten, die auf der Suche nach Arbeit von einem Land ins andere weiterziehen. Die Tatsache, dass drei verschiedene Kategorien von Personen betroffen sind, erschwert eine einheitliche Messung des Phänomens, dies umso mehr, als zahlreiche Geld- und Gütertransfers (zwischen 10 und 50 Prozent aller Fälle) über informelle Kanäle abgewickelt werden und damit nicht gemessen werden können. Siehe S. Puri und T. Ritzema, Migrant Worker Remittances, Micro-Finance and the Informal Economy: Prospects and Issues, Working Paper, Nr. 21, Social Finance Unit, Geneva, International Labour Organization, 1989. 
sich ihre Wirkung korrekt abschätzen. Die zur Messung der Finanzströme verwendeten Daten - handle es sich um Statistiken zur Zahlungsbilanz oder um Umfrageergebnisse - vermögen indessen kaum die Gesamtheit der Rückführungsmöglichkeiten abzudecken. Ein weiteres Hindernis ist schliesslich die Tatsache, dass die Transfers längst nicht immer über formelle, in die offiziellen Erhebungen einfliessende Kanäle abgewickelt werden. Tatsächlich sind in gewissen Kontexten informelle Transfers die Regel.

Mehrere internationale Organe haben in der Zwischenzeit begonnen, das Phänomen der Rücküberweisungen quantitativ zu erfassen, und es liegen verschiedene Schätzungen vor. Die detaillierteste und aussagekräftigste dürfte jene des IFAD sein, auf die im Folgenden Bezug genommen wird ${ }^{4}$. Der IFAD, der jedes Jahr statistische Daten publiziert, schätzte für das Jahr 2006 die Anzahl der Rücküberweisungen in Schwellen- und Entwicklungsländer auf 1,5 Milliarden (d. h. rund zehn Sendungen pro Migrantin und Migrant) und das Gesamtvolumen auf 300 Milliarden Dollar. In diesem Betrag nicht berücksichtigt sind Transfers zwischen Industrieländern, die ebenfalls ein bedeutendes Volumen aufweisen, sowie Geldströme zwischen Migrantinnen und Migranten, die im selben Gaststaat leben. Diese Zahlen sind bedeutend höher als die Schätzungen der OECD für das Jahr 2002, die die formellen Rücküberweisungen mit insgesamt 149,4 Milliarden Dollar bezifferte.

Selbst wenn die verfügbaren Zahlen zurückhaltend zu interpretieren sind, so veranschaulichen sie doch die Wirkung der Migration auf die Einkommen in den Ländern des Südens. Die wichtigsten Empfängerländer solcher Transfers sind - ebenfalls laut IFAD - unbestrittenermassen die asiatischen Staaten mit insgesamt 114 Milliarden Dollar (davon entfallen allein 24,5 Milliarden auf Indien, 21 Milliarden auf China und 15 Milliarden auf die Philippinen), gefolgt von Lateinamerika (gesamthaft 68 Millionen Dollar, wovon allein 24 Milliarden nach Mexiko fliessen) und den mittel-, südost- und osteuropäischen Ländern mit 51 Millionen Dollar (wovon 14 Milliarden auf die Russische Föderation und 8,5 Milliarden auf die Ukraine entfallen) ${ }^{5}$. Die afrikanischen Länder finden sich weiter hinten auf der Rangliste. Sie empfangen schätzungsweise 39 Milliarden Dollar (wovon je mehr als 5 Milliarden nach Algerien, Marokko und Nigeria fliessen), dicht gefolgt vom Mittleren Osten mit insgesamt 30 Milliarden Dollar (wovon 7,5 Milliarden auf die Türkei und 5,5 Milliarden auf den Libanon entfallen). Mehr als 60 Länder auf der ganzen Welt empfangen jedes Jahr Rücküberweisungen im Umfang von mehr als einer Milliarde Dollar, und 19 afrikanische Staaten erhalten jährlich mehr als eine halbe Milliarde Dollar.

Bezogen auf die Einwohnerzahl erhält laut IWF Jamaika mehr als 500 Dollar pro Kopf ${ }^{6}$. Das wichtigste afrikanische Empfängerland ist Kap Verde mit 320 Dollar pro Einwohner, das ist dieselbe Summe wie für Kroatien. Gesamthaft betrachtet betragen die Geldtransfers nach Afrika weniger als 20 Dollar pro Kopf, verglichen mit 60 Dollar im Falle von Lateinamerika?

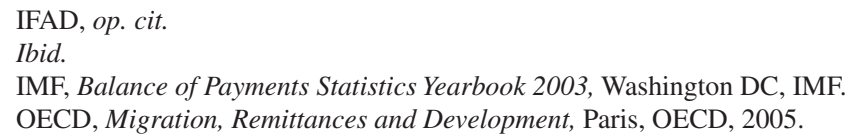


Im Verhältnis zum Bruttoinlandprodukt schliesslich erreichen die Transfers in rund 20 Ländern weltweit die Schwelle von 10 Prozent $^{8}$; in Moldawien, Jordanien, Kap Verde sowie Lesotho belaufen sie sich gar auf über 20 Prozent. Diese an sich schon beeindruckenden Zahlen sind umso bedeutender, als sich die ökonomische Wirkung der Transfers multipliziert: Fliesst Kapital in eine Volkswirtschaft, so erhöhen sich die Produktionskapazitäten um ein Mehrfaches, da das Geld unter den Wirtschaftsakteuren zirkuliert. Laut Durand, Parrado und Massey bewirkt jeder Dollar, der via Rücküberweisungen in die Volkswirtschaften des Südens geleitet wird, Ausgaben für Güter und Dienstleistungen im Umfang von 4 Dollar9 . Im konkreten Falle Mexikos schätzt Ratha den Multiplikationseffekt in städtischen Gebieten etwas zurückhaltender auf rund 2,69 und in ländlichen Gegenden auf 3,1710.

\section{Rücküberweisungen: Determinanten und Transferkanäle}

Das Verständnis der Rolle, die die Rücküberweisungen bei der Entwicklung der Länder des Südens spielen können, setzt voraus, dass die Beweggründe für das Senden von Geld in die Heimat bekannt sind ${ }^{11}$. Gewisse Autoren wie Straubhaar und Vadean sehen in diesen Transfers intuitiv das Ergebnis eines „altruistischen" Verhaltens beziehungsweise einer intrafamiliären Solidarität mit dem Ziel, die Lebensbedingungen der in der Heimat verbliebenen Familienangehörigen zu verbessern ${ }^{12}$. Zuweilen dienen die Rücküberweisungen auch zur Tilgung von Schulden, die die Familie zur Finanzierung des Studiums der ausgewanderten Person oder ihrer Auswanderung gemacht hat. Dieser Ansatz suggeriert das Vorhandensein eines familiären Drucks zur Auswanderung, die allerdings einen finanziellen Nutzen bringen soll. Rücküberweisungen können aber auch in einem persönlicheren Interesse erfolgen, beispielsweise zur Akkumulation von Gütern in der Heimat im Hinblick auf die Rückkehr, zum Aufbau einer Handelsoder Gewerbetätigkeit oder schlicht als wirtschaftliche Investitionen. In jedem Fall aber sind der Migrant und die in der Heimat verbliebenen Angehörigen nicht nur Akteure, sondern auch unmittelbar Begünstigte der Rücküberweisungen. Sie entscheiden auch über die Verwendung des transferierten Kapitals.

Diese starke familiäre oder individuelle Dimension deutet darauf hin, dass die Anzahl und die Höhe der Rücküberweisungen sowohl von den nach der Auswanderung bestehenden familiären Bindungen als auch von den Lebensumständen der in der Heimat verbliebenen Angehörigen abhängen. Je schlechter die

8 Tonga, Cisjordanien und Gaza, Lesotho, Jordanien, Kap Verde, Moldawien, Vanuatu, Bosnien und Herzegowina, Guyana, Jamaika, Albanien, Mazedonien, Nicaragua, El Salvador, Jemen, Dominikanische Republik, Ghana, Armenien und Honduras (IMF, op. cit.).

9 J. E. Durand, A. Parrado und D. S. Massey, ,Migradollars and Development: A Reconsideration of the Mexican Case“, International Migration Review, Bd. 30, Nr. 2, 1996, S. 423-444. Siehe auch J. E. Taylor, ,The new Economics of Labour Migration and the Role of Remittances in the Migration Process“, International Migration, Bd. 37, Nr. 1, 1999, S. 63-89.

10 D. Ratha, „Workers' Remittances: An Important and Stable Source of External Development Finance“, in World Bank, Global Development Finance 2003, Washington, DC, The World Bank, S. $157-175$.

11 Eine Übersicht über die verschiedenen Erklärungsansätze findet sich in OECD, op. cit.

12 T. Straubhaar und F. P. Vadean, ,Introduction. International Migrant Remittances and their Role in Development", in Organisation for Economic Co-operation and Development (OECD), Migration, Remittances and Development, Paris, OECD, 2005. 
Lebensbedingungen der Familie im Herkunftsland, desto höher die Wahrscheinlichkeit, dass diese Zahlungen aus dem Ausland erhält, und desto grösser die Gefahr einer wirtschaftlichen Abhängigkeit von solchen Rücküberweisungen. Umgekehrt nimmt die Notwendigkeit der Transfers ab, wenn sich die wirtschaftliche Situation des Haushalts verbessert oder das Gefälle zwischen den Lebensbedingungen der Migranten und der begünstigten Haushalte abflacht.

Dies lässt sich auch auf der Ebene der lokalen Gemeinschaften feststellen, deren Entwicklungsstand die Höhe und Anzahl der erhaltenen Transfers beeinflussen kann. Bei Armut oder in politischen, gesundheitlichen oder wirtschaftlichen Krisensituationen werden die im Ausland lebenden Angehörigen mobilisiert, während im umgekehrten Fall die Rücküberweisungen abnehmen können. Remittances weisen demnach einen antizyklischen Charakter auf, denn sie nehmen in wirtschaftlichen Notsituationen zu.

Auch der Verlauf der Migration kann das Verhalten der Migrantinnen und Migranten beeinflussen. So wurde insbesondere nachgewiesen, dass der Wunsch oder die Absicht, in das Herkunftsland zurückzukehren, für die Rücküberweisungspraxis bestimmend sind ${ }^{13}$. Ausserdem nimmt der Umfang der Rücküberweisungen in der Regel mit zunehmender Aufenthaltsdauer im Gastland ab. Ein massgeblicher Faktor ist ferner die Art der Migration, das heisst, ob eine einzelne Person oder eine ganze Familie auswandert.

Damit zeigt sich, dass die Migrationspolitiken einen unmittelbaren Einfluss auf die Rücküberweisungen haben können. In diesem Zusammenhang weisen Dumont und Faini insbesondere darauf hin, dass der selektive Charakter der heutigen Migrationspolitiken der westlichen Länder, welche die Einwanderung hoch qualifizierter Arbeitskräfte fördern soll, ohne Zweifel Folgen auf den Umfang der Rücküberweisungen haben wird ${ }^{14}$. Tatsächlich dürfte ein hoch qualifizierter Migrant allein aufgrund des Gehaltsunterschiedes und der unterschiedlichen Erwartungen an die Migration andere Rücküberweisungsstrategien verfolgen als ein unqualifizierter Einwanderer. Allerdings kommen Untersuchungen zu dieser Frage noch immer zu widersprüchlichen Ergebnissen; für ein besseres Verständnis sind vertiefte Analysen somit unverzichtbar.

Migranten, die Rücküberweisungen im Hinblick auf ihre Rückkehr in die Heimat tätigen, orientieren sich dabei an der finanziellen Lage im Herkunftsland. Sind Investitionsmöglichkeiten vorhanden - Kennzahlen dafür sind das Zinsniveau, die Inflation, die Renditemöglichkeiten usw. -, erhöhen sich tendenziell nicht nur die überwiesenen Beträge, sondern auch deren Wirkungen auf die wirtschaftliche Entwicklung des Landes.

Die rein private Natur der Rücküberweisungen sowie ihr wichtigster Zweck nämlich die Lebensumstände der Haushalte im Herkunftsland zu verbessern bewirken, dass regelmässig (häufig monatlich) relativ kleine Summen in die Heimat gesandt werden. So zeigt eine in der Schweiz durchgeführte Umfrage, dass serbischstämmige Migrantenhaushalte jedes Jahr im Median 3000 Schweizer Franken verteilt auf mehrere Zahlungen in das Herkunftsland überweisen. Dieser Betrag weicht nur geringfügig vom Ergebnis einer amerikanischen Studie ab, die

13 D. J. Conway and H. Cohen, „Consequences of Migration and Remittances for Mexican Transnational Communities“, Economic Geography, Bd. 74, Nr. 1, 1998, S. $26-44$.

14 J.-C. Dumont, „Migrations et transferts de fonds. Impact sur les pays d'origine. Commentaires“, Revue d'économie du développement, Bd. 21, Nr. 2-3, 2007, S. 189-194. R. Faini, op. cit. 
zum Schluss kam, dass lateinamerikanische Migranten im Durchschnitt jedes Jahr ungefähr 3000 Dollar in die Heimat schicken. Dabei sind zwischen den Nationalitäten Unterschiede zu erkennen: Mexikaner beispielsweise überweisen mit monatlich knapp 400 Dollar (bzw. 22 Prozent ihres Einkommens) doppelt so viel Geld wie Einwanderer aus der Dominikanischen Republik ${ }^{15}$.

Welche Kanäle für die Rücküberweisungen genutzt werden, hängt nicht nur von diesen Determinanten, sondern auch von der Struktur des Bankenwesens im Gast- und insbesondere im Empfängerland ab. Da in den Ländern des Südens und namentlich in den ländlichen Gebieten das Bankenwesen wenig entwickelt ist, erfolgen die Rücküberweisungen über spezifische Finanzakteure, angefangen von grossen Institutionen wie Western Union mit weltweit 170000 Zweigstellen bis hin zu Kleinstdienstleistern wie gewisse ethnic stores, die sich gezielt auf eine einzelne Gruppe von Migranten konzentrieren. Neben diesen Intermediären, die auf das Geschäft mit Rücküberweisungen von Migrantinnen und Migranten spezialisiert sind, spielen Banken und Postunternehmen nur in jenen Ländern eine wichtige Rolle, in denen das Zweigstellennetz ausreichend dicht und die Tarife attraktiv sind. Der Hauptgrund für die Zurückhaltung, mit der die Banken nach und nach im Rücküberweisungsgeschäft Fuss fassen, ist das Fehlen einer Infrastruktur in den Empfängerländern. Daneben spielen aber auch Sicherheitsaspekte eine Rolle, denn zuweilen werden Rücküberweisungen mit Terrorismusfinanzierung oder Geldwäscherei in Verbindung gebracht.

Angesichts dessen überrascht es nicht, dass ein bedeutender Anteil dieser Transfers - je nach Land bis zu 80 Prozent - über informelle Kanäle abgewickelt werden. Diese Kanäle sind unterschiedlich strukturiert und unter verschiedenen Bezeichnungen bekannt; Beispiele dafür sind das Hawala-System der Migranten aus Südasien und dem Mittleren Osten oder Hundi, das von indischen Auswanderern genutzt wird. Diese Systeme arbeiten in der Regel in länderübergreifenden Netzen: Ein Intermediär aus der Gemeinschaft im Gastland nimmt das Geld entgegen und gibt einem Intermediär im Herkunftsland den Auftrag, den entsprechenden Betrag an den Begünstigten auszuzahlen. Die Kompensationsmechanismen zwischen den beiden Intermediären gewährleisten das einwandfreie Funktionieren dieser Überweisungsart, die ebenso schnell ist wie formelle Kanäle, aber häufig günstiger.

Zuweilen werden aber auch weniger sichere Kanäle genutzt. Für die Migranten besteht dabei häufig ein grosses Risiko, dass das Geld unterschlagen wird. Hierzu zählen beispielsweise der Versand von Bargeld auf dem Postweg oder der physische Transfer über einen Mittelsmann. Diese Praktiken profitieren von den hohen Tarifen der Finanzinstitute für Rücküberweisungen und vom mangelnden Vertrauen in die Bankensysteme. Selbst nach Ländern mit einem relativ dichten Bankennetz wird ein beachtlicher Anteil der Transfers über Mittelsmänner abgewickelt. Laut einem Bericht werden 10 Prozent aller nach Lateinamerika überwiesenen Beträge auf diese Weise überbracht, während Bargeldsendungen auf dem Postweg rund 5 Prozent ausmachen dürften ${ }^{16}$.

15 M. Orozco, Remittances to Latin America and the Caribbean: Issues and Perspectives on Development, Report Commissioned by the Office for the Summit Process, Washington, DC, Organization of American States, September 2004.

16 R. Suro, S. Bendixen, B. L. Lowell und D. C. Benavides, Billions in Motion: Latino Immigrants, Remittances and Banking, Washington, DC, Pew Hispanic Center; Multilateral Investment Fund, 2002. 
Mit Ausnahme gewisser lateinamerikanischer Länder wie Guatemala, Honduras und Ecuador, in denen Banken solide verankert und für mehr als 30 Prozent der Bevölkerung zugänglich sind ${ }^{17}$, werden die meisten überwiesenen Beträge ohne Umweg über ein Bankkonto direkt an die Begünstigten ausbezahlt. Tatsächlich erfordert das Überweisen von Geld auf dem Bankenweg, dass sowohl der sendende Migrant als auch der Begünstigte über ein Konto verfügen. Dies ist jedoch längst nicht immer der Fall, sei es aufgrund des rechtlichen Status des Migranten im Gastland (illegaler Aufenthalt) oder der zu grossen Entfernung, weil in der näheren Umgebung keine Bankfiliale vorhanden ist.

Diese unterschiedlichen Organisationsformen können die Höhe der überwiesenen Beträge und ihre Verwendung beeinflussen. Je nach gewähltem Kanal kann der an die Familien ausbezahlte Anteil des Betrags variieren. Dabei ist zu präzisieren, dass sich im Zuge des technologischen Fortschritts die effektiven Kosten einer Überweisung verringern und gleichzeitig die Abwicklung beschleunigt wird. Die Tarife der Finanzorganismen dagegen orientieren sich eher an den Marktgesetzen als an den effektiven Kosten. Die inoffiziellen Überweisungsmethoden wie Hawala sind relativ günstig (zwischen 1,5 und 2 Prozent des Überweisungsbetrags) ${ }^{18}$, während bei den formellen Kanälen sehr hohe Gebühren anfallen, die mehr als 10 Prozent des Überweisungsbetrags erreichen können.

Die Höhe dieser Gebühren wurde von zahlreichen Beobachtern kritisiert. In der Schweiz forderte Nationalrat Carlo Sommaruga in seinem Postulat vom 4. Oktober 2005 die Einführung von Kostenbegrenzungsmechanismen. Der Vorstoss wurde mit dem Argument abgelehnt, die Zahl der in der Schweiz tätigen Anbieter sei ausreichend gross, um den freien Wettbewerb sicherzustellen, der seinerseits dafür sorgen würde, dass sich die Gebühren dem Niveau der effektiven Kosten annähern. Diese Behauptung ist jedoch insofern zu relativieren, als die Situation im Empfängerland - das heisst das Vorhandensein von Zweigstellen in der Wohnregion der Begünstigten - eine mindestens ebenso wichtige Rolle spielt wie jene im Gastland.

Das Beispiel der Vereinigten Staaten zeigt allerdings, dass die Gebühren unter echten Wettbewerbsbedingungen tatsächlich sinken können. Zwischen 1990 und 2002 hat sich die Zahl der Finanzinstitute mit Niederlassungen sowohl in den USA als auch im Herkunftsland im Falle von Mexiko von drei auf acht und für die Dominikanische Republik von drei auf sechs erhöht. In der Folge sanken die Überweisungsgebühren in bedeutendem Umfang von 15 auf 5 Prozent des Überweisungsbetrags ${ }^{19}$.

\section{Wirkungen der Rücküberweisungen auf die wirtschaftliche und soziale Entwicklung der Empfängerländer}

Um die Wirkungen der Rücküberweisungen adäquat beurteilen zu können, müsste idealerweise ein Vergleich gezogen werden zwischen der Situation mit

17 M. Orozco, op. cit.

18 OECD, op. cit.

19 M. Orozco, Workers' Remittances: Issues and Best Practices, Testimony presented to the US House Committee on Financial Services hearing entitled „Remittances: Reducing Costs, Increasing Competition, and Broadening Access to the Market“, 1. Oktober 2003. 
beziehungsweise ohne den Zufluss von Remittances. Allerdings liegt bis heute kein Modell vor, das einen solchen Vergleich ermöglichen würde, und die Literatur beschränkt sich in der Regel auf die Beschreibung und Interpretation der in den Auswanderungsländern empirisch beobachteten Wirkungen ${ }^{20}$, ohne jedoch die damit verbundenen Kosten (Abwanderung qualifizierter Fachkräfte, Ungleichgewicht auf dem Arbeitsmarkt usw.) zwingend zu berïcksichtigen. Die beobachteten Erfahrungen lassen aber darauf schliessen, dass der Zufluss von Rücküberweisungen die wirtschaftliche und soziale Entwicklung der Empfängerländer positiv beeinflusst.

Es gilt als erwiesen, dass die Wirkung der Rücküberweisungen auf die Entwicklung davon abhängt, wie diese Gelder verwendet werden. Dabei wird von der Hypothese ausgegangen, dass sie ihren grössten Nutzen entfalten, wenn sie für Investitionen in die Wirtschaftstätigkeit verwendet werden. Allerdings hat eine neuere Studie der Weltbank in sechs Staaten der ehemaligen UdSSR beispielsweise gezeigt, dass 10 bis 15 Prozent der zufliessenden Gelder in das Humankapital (Ausbildung) und nur ein marginaler Anteil von rund 5 Prozent in die Wirtschaft investiert werden ${ }^{21}$. Die verbleibenden 80 Prozent fliessen in den Konsum, und zwar in erster Linie in den Erwerb von Grundgütern. Ähnliche Anteile wurden bei den lateinamerikanischen Haushalten beobachtet, die Geldsendungen aus den Vereinigten Staaten erhalten ${ }^{22}$. Besonders hoch ist der Anteil der für Konsumgüter aufgewendeten Beträge bei Empfängerhaushalten mit niedrigem Lebensstandard. Hier tragen die Rücküberweisungen in erster Linie zur Linderung der Armut bei. Wie bereits erwähnt, weist diese „Versicherungsfunktion" der Auswanderer, die Geld in die Heimat schicken, antizyklische Merkmale auf, das heisst, die überwiesenen Beträge steigen, wenn die in der Heimat zurückgebliebenen Familienmitglieder von wirtschaftlichen oder politischen Krisen betroffen sind.

Verschiedene Risiken im Zusammenhang mit diesen Transfers wurden nachgewiesen und müssen bei einer allgemeinen Erörterung ihrer Wirkung berücksichtigt werden ${ }^{23}$. Insbesondere kommen nicht alle Haushalte in den Genuss von Geldsendungen aus dem Ausland, und verschiedentlich wurde die Befürchtung geäussert, dass sich die Ungleichheit zwischen jenen, die Rücküberweisungen erhalten, und den anderen, die ohne solche Gelder auskommen müssen, verstärken könnte. Dies ist beispielsweise in Pakistan der Fall, wo nur die sozial am besten gestellten Haushalte in der Lage sind, die Auswanderung eines ihrer Angehörigen zu finanzieren und im Gegenzug allenfalls einen Nutzen daraus zu ziehen $^{24}$. In Albanien haben die Transfers das Gefälle zwischen armen und reichen Regionen verstärkt: Letztere ziehen interne Migranten an und beherbergen auch Familien von im Ausland lebenden Migranten ${ }^{25}$. In Mexiko dagegen weisen die Bewohnerinnen und Bewohner der ärmsten Regionen die höchste Wahr-

20 Siehe zum Beispiel OECD, op. cit.

${ }_{21}$ World Bank, Migration and Remittances: Eastern Europe and the Former Soviet Union, Washington, DC, The World Bank, 2006.

22 M. Orozco, Remittances to Latin America and the Caribbean, op. cit.

23 J.-C. Dumont, op. cit.

24 R. H. Adams, „Remittances, Investment and Rural Asset Accumulation in Pakistan“, Economic Development and Cultural Change, Bd. 47, Nr. 1, Oktober 1998, S. 155-173.

25 M. Lerch, Les transferts de fonds des migrants albanais. Facteurs déterminant leur réception, Neuchâtel, Forum suisse pour l'étude des migrations et de la population, 2006. 
scheinlichkeit auf, ins Ausland zu migrieren ${ }^{26}$. Auch auf Länderebene ist eine zunehmende Ungleichheit bei der Verteilung der Rücküberweisungen feststellbar: Vor allem die Schwellenländer wie Indien und China kommen in den Genuss von Rücküberweisungen, während die ärmsten Länder nur marginal davon profitieren.

Ganz abgesehen von der Gefahr einer zunehmenden Ungleichheit kann sich auch die von den Rücküberweisungen hervorgerufene wirtschaftliche Abhängigkeit negativ auswirken. Noch vor wenigen Jahren standen die Rücküberweisungen häufig im Ruf, Neid zu schüren und berufliche Gewohnheiten erodieren zu lassen, wie Keeley und Tran mit dem Zitat von Russell belegen ${ }^{27}$. In jüngerer Zeit vertrat Gedeshi im Hinblick auf Albanien den Standpunkt, wonach Migration als unverzichtbare Voraussetzung zur Erlangung eines als akzeptabel geltenden Lebensstandards betrachtet werden $\mathrm{kann}^{28}$. Verschiedene Autoren haben zudem nachgewiesen, dass der Erhalt von Geldsendungen aus dem Ausland zu einer Abnahme der Erwerbstätigkeit in den begünstigten Haushalten führt, denn diese sind wenig geneigt, für einen geringen Lohn arbeiten $\mathrm{zu}$ gehen, wenn das Geld aus dem Ausland das Überleben sichert ${ }^{29}$.

Am schärfsten kritisiert werden indessen das Konsumverhalten und die wenig adäquaten Investitionen der Begünstigten. Ihnen wird häufig vorgeworfen, nicht in Tätigkeiten zu investieren, die Arbeitsplätze oder Wohlstand schaffen, und so gegen die „Spielregeln“ der Entwicklung zu verstossen. Aber wie liesse sich die Verwendung der aus der Migration stammenden Ressourcen ändern? Die Frage ist nach wie vor offen, und Bestrebungen, einen Teil der Transfers zur wirtschaftlichen und sozialen Entwicklung und zum Ausbau der Infrastrukturen zu nutzen, beschränken sich zumeist auf einige wenige lokale Vorstösse mit mässigem Erfolg.

Auf makroökonomischer Ebene besteht der Hauptnutzen der Rücküberweisungen in der Beschaffung von Devisen, die mitunter für den Ausgleich der Zahlungsbilanz benötigt werden. Ausserdem schaffen sie Investitionsmöglichkeiten, deren Wirkungen allerdings nicht immer positiv sind: So wurden beispielsweise in gewissen Kontexten aufgrund der Zunahme der finanziellen Rückflüsse aus der Migration inflationäre Tendenzen festgestellt ${ }^{30}$. Nicht zuletzt können diese Transfers, wenn sie in die Wirtschaft investiert werden, die Regierungen dazu verleiten, ihre eigenen Investitionen zurückzustellen ${ }^{31}$.

26 O. Stark und J. E. Taylor, „Relative Deprivation and International Migration“, Demography, Bd. 26, Nr. 1, 1989, S. 1-14.

27, ,...] remittances are accused of creating envy and eroding work habits “ (S. S. Russell, „Remittances from International Migration: A Review in Perspective“, World Development, Bd. 14, Nr. 6, 1986, S. 678, zitiert in: C. B. Keely und Bao Nga Tran, „Remittances from Labor Migration: Evaluations, Performance and Implications“, International Migration Review, Bd. 23, Nr. 3, 1989, S. 502).

28 I. Gedeshi, „Role of Remittances from Albanian Emigrants and Their Influence in the Country's Economy“, Eastern European Economics, Bd. 40, Nr. 5, 2002, S. 49-72.

29 Siehe zum Beispiel R. Chami, C. Fullenkamp und S. Jahjah, Are Immigrant Remittance Flows a Source of Capital for Development?, Working Paper, Nr. 03/189, Washington, DC, International Monetary Fund (IMF), 2003.

30 Siehe OECD, op. cit. Als Beispiel werden die aufgrund zunehmender Nachfrage steigenden Bodenpreise in Ägypten angeführt.

31 N. P. Glytsos, „The Role of Migrant Remittances in Development: Evidence from Mediterranean Countries", International Migration, Bd. 40, Nr. 1, 2002, S. 5-26. 
Obwohl sie ein grosses Potenzial besitzen, tragen Rücküberweisungen in den ärmsten Ländern nicht immer optimal zur Entwicklung bei. Auf der Ebene der Staaten zeigt sich, dass vor allem gewisse Schwellenländer mit einem hohen Anteil an Auswanderern in den Genuss von Rücküberweisungen kommen. Aber selbst in diesen Ländern wird nicht das gesamte Potenzial dieser Transfers ausgeschöpft, und die Ungleichheiten zwischen Begünstigten und Nichtbegünstigten können sich verstärken.

Es wurden zahlreiche Initiativen lanciert mit dem Ziel, die Situation zu verbessern. Ihre Möglichkeiten sind jedoch begrenzt, da die Rücküberweisungen zwischen Privaten abgewickelt werden, die über die Verwendung der Gelder frei entscheiden können. Der Einsatz der Gelder lässt sich somit nur durch starke Anreize kanalisieren. Verschiedene Best-Practice-Beispiele haben gezeigt, dass mit Mischfinanzierungen zuweilen ansprechende Resultate erzielt werden können $^{32}$. Ein Beispiel hierfür ist das Programm „Tres Por Uno“ aus den späten 1980er-Jahren, in dessen Rahmen für jeden Dollar, der von Migranten in Projekte von öffentlichem Interesse investiert wurde, drei Dollar von lokalen, regionalen und nationalen Behörden bereitgestellt wurden.

Insbesondere macht die Analyse der Rücküberweisungen aber auch deutlich, dass angemessenere Migrationspolitiken der Länder des Nordens zweifellos zur Bekämpfung der Armut in den Ländern des Südens beitragen könnten. Diese Politiken beruhen heute auf Selektionskriterien, die die Einwanderung hoch qualifizierter Arbeitskräfte und ihrer Familien begünstigen. Aus diesem Grund ist die Wahrscheinlichkeit, dass eine Person aus einem einkommensschwachen Land in ein Industrieland auswandert, geringer als für Migrationswillige aus Schwellenländern, in denen das Bildungsniveau höher ist und damit die Auswanderungsmöglichkeiten zahlreicher sind. Als Folge davon profitieren von der Migration in erster Linie Länder wie Indien und China, die über gute Bildungsinfrastrukturen verfügen, welche die Auswanderungsmöglichkeiten erhöhen, während die ärmsten Länder, beispielsweise jene in Subsahara-Afrika, einen weitaus geringeren Nutzen daraus ziehen. Migration und Entwicklung miteinander in Einklang zu bringen würde voraussetzen, dass die Auswanderungsmöglichkeiten für Menschen aus diesen Ländern, die bis heute in der internationalen Migration eine Nebenrolle spielen, hinterfragt werden. Die heute in den Ländern des Nordens vorherrschenden Migrationspolitiken sind davon allerdings weit entfernt.

Eine weitere wichtige Voraussetzung dafür, dass die Migration zur Verwirklichung der Entwicklungsziele beitragen kann, ist, dass die Bindungen zwischen Migrant und Herkunftsland um jeden Preis aufrechterhalten werden. Dies ist der Fall, wenn die Migration zeitlich befristet ist, das heisst, wenn sie mit dem festen Vorsatz verbunden ist, nach einigen Jahren in die Heimat zurückzukehren, oder wenn regelmässige und enge Kontakte mit dem Herkunftsland gepflegt werden. In diesem Fall kann die Migration am ehesten gewährleisten, dass erstens die Rücküberweisungen in die Heimat zunehmen und zweitens ein Austausch von Humankapital zwischen den Ländern stattfindet. Um Migration

32 Siehe OECD, op. cit. 
und Entwicklung miteinander in Einklang zur bringen, müssen zudem Massnahmen geprüft werden, die auch für die ärmsten Länder eine gewisse Form von Personenfreizügigkeit gewährleisten. Nach wie vor prioritär sind schliesslich auch Massnahmen zur Senkung der Überweisungsgebühren und zur Erhöhung der Sicherheit von Geldsendungen.

\section{Bibliographie}

Adams, R. H., „Remittances, Investment and Rural Asset Accumulation in Pakistan“, Economic Development and Cultural Change, Bd. 47, Nr. 1, Oktober 1998, S. 155-173.

Chami, R., C. Fullenkamp and S. Jahjah, Are Immigrant Remittance Flows a Source of Capital for Development?, Working Paper, Nr. 03/189, Washington, DC, International Monetary Fund (IMF), 2003.

Conway, D. J. and H. Cohen, „Consequences of Migration and Remittances for Mexican Transnational Communities“, Economic Geography, Bd. 74, Nr. 1, 1998, S. 26-44.

Dumont, J.-C., „Migrations et transferts de fonds. Impact sur les pays d'origine. Commentaires“, Revue d'économie du développement, Bd. 21, Nr. 2-3, 2007, S. 189-194.

Durand, J. E., A. Parrado and D. S. Massey, „Migradollars and Development: A Reconsideration of the Mexican Case“, International Migration Review, Bd. 30, Nr. 2, 1996, S. 423-444.

Faini, R., „Migrations et transferts de fonds. Impact sur les pays d'origine“, Revue d'économie du développement, Bd. 21, Nr. 2-3, 2007, S. 153-182.

International Monetary Fund (IMF), Balance of Payments Statistics Yearbook 2003, Washington DC, IMF.

Gedeshi, I., „Role of Remittances from Albanian Emigrants and Their Influence in the Country's Economy“, Eastern European Economics, Bd. 40, Nr. 5, 2002, S. 49-72.

Glytsos, N. P., „The Role of Migrant Remittances in Development: Evidence from Mediterranean Countries“, International Migration, Bd. 40, Nr. 1, 2002, S. 5-26.

Gubert, F., „Migrations et transferts de fonds. Impact sur les pays d'origine. Commentaires“, Revue d'économie du développement, Bd. 21, Nr. 2-3, 2007, S. 183-188.

International Fund for Agricultural Development (IFAD), Sending Money Home, Rome, IFAD, Dezember 2007, <http://www.ifad.org/events/remittances/maps/brochure.pdf>.

Keely, C. B. und Bao Nga Tran, „Remittances from Labor Migration: Evaluations, Performance and Implications", International Migration Review, Bd. 23, Nr. 3, 1989, S. 500-525.

Lerch, M., Les transferts de fonds des migrants albanais. Facteurs déterminant leur réception, Neuchâtel, Forum suisse pour l'étude des migrations et de la population, 2006.

Organisation for Economic Co-operation and Development (OECD), Migration, Remittances and Development, Paris, OECD, 2005.

Orozco, M., Remittances to Latin America and the Caribbean: Issues and Perspectives on Development, Report Commissioned by the Office for the Summit Process, Washington, DC, Organization of American States, September 2004.

Orozco, M., Workers' Remittances: Issues and Best Practices, Testimony presented to the US House Committee on Financial Services hearing entitled „Remittances: Reducing Costs, Increasing Competition, and Broadening Access to the Market“, 1. Oktober 2003.

Puri, S. und T. Ritzema, Migrant Worker Remittances, Micro-Finance and the Informal Economy: Prospects and Issues, Working Paper, Nr. 21, Social Finance Unit, Geneva, International Labour Organization, 1989.

Ratha, D., „Workers' Remittances: An Important and Stable Source of External Development Finance“, in World Bank, Global Development Finance 2003, Washington, DC, The World Bank, S. 157-175.

Russel, S. S., „Remittances from International Migration: A Review in Perspective“, World Development, Bd. 14, Nr. 6, 1986, S. 677-696.

Stark, O., und J. E. Taylor, „Relative Deprivation and International Migration“, Demography, Bd. 26, Nr. 1, 1989, S. 1-14.

Straubhaar, T. and F. P. Vadean, ,Introduction. International Migrant Remittances and their Role in Development", in Organisation for Economic Co-operation and Development (OECD), Migration, Remittances and Development, Paris, OECD, 2005.

Suro, R., S. Bendixen, B. L. Lowell and D. C. Benavides, Billions in Motion: Latino Immigrants, Remittances and Banking, Washington, DC, Pew Hispanic Center; Multilateral Investment Fund, 2002. 
Taylor, J. E., „The new Economics of Labour Migration and the Role of Remittances in the Migration Process", International Migration, Bd. 37, Nr. 1, 1999, S. 63-89.

World Bank, International Conference on Migrant Remittance: Development Impact, Opportunities for the Financial Sector and Future Prospects: Report and Conclusions, London, Department for International Development; The World Bank; International Migration Policy Programme, 2003.

World Bank, Migration and Remittances: Eastern Europe and the Former Soviet Union, Washington, DC, The World Bank, 2006. 